\title{
Desigualdades sociales en salud: Mortalidad en menores de cinco años, Honduras, 2014
}

\author{
Social inequalities in health: Mortality in children under five years, Honduras, 2014
}

Edith Geraldina González González, ${ }^{1}$ Nora Concepción Rodríguez Corea. ${ }^{2}$

${ }^{1}$ Licenciada en Enfermería, Maestría en Salud Pública; Departamento de Enfermería, Facultad de Ciencias Médicas (FCM), Universidad Nacional Autónoma de Honduras (UNAH) Valle de Sula, San Pedro Sula.

${ }^{2}$ Médico Especialista en Pediatría, Maestría en Epidemiología; Posgrado en Salud Pública, Departamento de Salud Pública, Facultad de Ciencias Médicas, Universidad Nacional Autónoma de Honduras, Tegucigalpa.

RESUMEN. Antecedentes: Las desigualdades sociales en salud se refieren a disparidades de salud, que se consideran injustas, evitables e innecesarias y que sistemáticamente recaen sobre poblaciones vulnerables. Objetivo: Medir las desigualdades sociales en salud de la mortalidad en los niños menores de cinco años, Francisco Morazán y Yoro, Honduras, año 2014. Métodos: Estudio descriptivo transversal. Se analizó la mortalidad de menores de 5 años a través de la medición de desigualdades sociales; se estimó las métricas de desigualdad en salud, índice de Kuznets relativo y absoluto, índice de desigualdad de la pendiente, índice de concentración en salud; se describió la situación de las desigualdades sociales en salud de la mortalidad por estratificador de equidad social (alfabetismo). Resultados: En 27 municipios del departamento de Francisco Morazán se registraron 71 menores de cinco años fallecidos, y en los 11 municipios del departamento de Yoro 131. Se encontró un exceso de 18 muertes de menores de cinco años por 1000 nacidos vivos entre la población con menor alfabetismo en Francisco Morazán, y de 23 en Yoro, en comparación con los municipios de mayor alfabetismo. En ambos departamentos la curva de concentración de la salud se mantuvo sobre la línea de equidad, concluyendo que la mortalidad de menores de cinco años se concentró en la población menos alfabetizada. Discusión: Se observó que los municipios con tasas más altas de alfabetismo presentaron menos mortalidad de menores de cinco años.

Palabras Clave: Desigualdades en salud, Mortalidad, índice de Concentración de desigualdad.
Recibido: 15-07-2020 Aceptado para publicación 17-12-2020

Dirección para correspondencia: Lic. Edith G. González

Correo electrónico: edith.gonzalez@unah.edu.hn

Declaración de relaciones y actividades financieras y no financieras y conflictos de interés: ninguno.

DOl:https://doi.org/10.5377/rmh.v88i2.11487

\section{INTRODUCCIÓN}

Desigualdad en salud es un término utilizado para referirse a cualquier diferencia en la salud de las poblaciones, es decir a la distribución de enfermedades, causas de muerte, factores de riesgo y otros aspectos relacionados con la salud que pueden ser evitadas. Las desigualdades en salud se definen como diferencias observables en la salud entre dos o más grupos socialmente determinados. Estas diferencias pueden objetivarse a partir de la observación simple y precisa de datos relevantes; por ejemplo, la mortalidad infantil es más alta en niños pobres que en ricos. ${ }^{1}$ En el año 2015 el Banco Mundial realizó un informe basado en datos con medición de desigualdad utilizando el índice de Gini que básicamente mide el ingreso económico; y ubica a Honduras en el sexto lugar a nivel mundial y primero en América Latina con mayor índice de desigualdad. ${ }^{2}$

La desigualdad en salud es el criterio de medición que permite valorar la inequidad en salud y tiene el propósito específico de servir de sustento a las políticas, los programas y las prácticas para reducir las diferencias en salud que se juzgan como arbitrarias e injustas. ${ }^{3}$ Los datos sobre las desigualdades en salud son la base para incorporar la equidad en la planificación de salud basada en la evidencia, así como para valorar si las iniciativas actuales en el ámbito de la salud promueven 0 no la equidad. La desigualdad es un concepto complejo que se puede medir y expresar usando una variedad de técnicas estadísticas. Cuando se mide la desigualdad en salud el propósito es siempre proporcionar una estimación cuantitativa de la desigualdad en salud en una población. ${ }^{4}$

Los estudios de desigualdades provienen casi totalmente de los países desarrollados, principalmente de Europa, la mayor parte de los estudios han sido publicados en Holanda, Suecia, Finlandia, y también en Estados Unidos a partir de finales de los $80,5,6$ y actualmente se carece de un análisis de desigualdades socioeconómicas de la Región de las Américas en su conjunto. ${ }^{7,8}$

Este estudio se realizó con el objetivo de medir y analizar las desigualdades sociales de la mortalidad de los niños (as) menores de 5 años a través de su medición, en los departa- 
mentos de Francisco Morazán y Yoro en Honduras durante el año 2014. Se considera fundamental que en la salud pública de Honduras se conozcan las desigualdades sociales en salud a nivel municipal para evidenciar y estudiar dichas diferencias injustificadas en la población y que la Secretaría de Salud y la academia realicen monitoreo de éstas.

\section{METODOLOGÍA}

La investigación realizada fue cuantitativa, el tipo de estudio descriptivo, de corte transversal. El área geográfica de estudio fueron 27 municipios del departamento de Francisco Morazán (se exceptuó el municipio del Distrito Central) y 11 municipios de Yoro. El universo y muestra lo constituyó la población de niños menores de cinco años que fallecieron y que estaban registrados en la base oficial de datos existentes en la Secretaria de Salud, correspondiendo al departamento de Francisco Morazán 71 niños fallecidos y 131 al departamento de Yoro durante el año 2014.

Se utilizó el estratificador social educación, enfocado en el índice de alfabetismo del año 2013 de la población mayor de 15 años de cada municipio, de ambos departamentos suministrado por la fuente pública: Instituto Nacional de Estadísticas (INE) $)^{9,10}$. Los criterios de inclusión comprendieron a todos los niños y niñas fallecidos menores de cinco años, de ambos departamentos del año en estudio, registrados en las bases de datos de estadísticas de la Secretaría de Salud: Se excluyeron los menores de cinco años fallecidos en el municipio del Distrito Central del departamento de Francisco Morazán y los fallecidos en otros departamentos.

El enfoque metodológico básico para la medición de las desigualdades en salud se centró en dos pasos: 1) comparación del estado de salud de dos o más grupos poblacionales socialmente definidos y 2) la expresión de esa comparación en una métrica-resumen, específica y estándar, de la desigualdad en salud. Esta métrica-resumen puede expresar una brecha (la diferencia entre dos y solo dos grupos sociales, usualmente extremos) o bien un gradiente (la magnitud de la desigualdad en salud a lo largo de toda la jerarquía social), y puede ser absoluta (expresada en las mismas unidades de la variable del estado de salud) o bien relativa (sin dimensión, solo indicando la desproporcionalidad). ${ }^{1}$

En este estudio los tres elementos centrales en la conceptualización del enfoque analítico para medir desigualdades sociales en salud de la mortalidad de los menores de cinco años se realizaron a través de las métricas de medición: Índice de Kuznets relativo y absoluto, Índice de desigualdad de la pendiente (como métrica de gradiente absoluto de desigualdad), e Índice de concentración de la desigualdad en salud. ${ }^{11,12}$

En la metodología de cálculo de las métricas de medición de desigualdades se utilizaron tres dimensiones requeridas para el análisis de desigualdad: 1) Un Indicador de salud (mortalidad de los niños menores de cinco años), 2) El estratificador de equidad (alfabetismo a nivel municipal, año 2013), 3) Variables demográficas denominadores de los indicadores de salud: la población menor de cinco años viva en ambos departamentos en 2014.

La fórmula utilizada para la tasa de mortalidad de los niños menores de cinco años fue la siguiente: Número de muertos menores de 5 años en (t) periodo de tiempo x 1000/ Número de menores de 5 años vivos en el periodo de tiempo.

Las métricas de mediciones simples de la desigualdad ${ }^{4,13}$ utilizadas fueron 1) Desigualdad absoluta: esta refleja la magnitud de la diferencia en salud entre dos grupos, 2) Desigualdad relativa: muestra las diferencias proporcionales en la salud entre grupos. Las Métricas de Mediciones complejas de desigualdad 4,12,13 utilizadas fueron 1) Índice de desigualdad de la pendiente: (métrica de regresión) representa la diferencia absoluta en los valores predichos de un indicador de salud entre aquellos con el nivel más bajo de educación o riqueza, tomando en cuenta la distribución integra de la educación o la riqueza usando un modelo de regresión apropiado; 2) Índice de concentración: (métrica de desproporcionalidad, esta métrica indica el grado en que se concentra un indicador de salud entre los más desaventajados o los más aventajados. La población se ordena de peor a mejor estado socioeconómico, el índice de concentración tiene un valor negativo cuando el indicador de salud se concentra en los más desaventajados. De no existir desigualdad, el índice de concentración es igual a cero.

Para el cálculo de la brecha absoluta y relativa se empleó la estadística descriptiva usando los percentiles: quintiles y cuartiles. La información por municipio se ordenó de menor nivel de alfabetismo a mayor nivel de alfabetismo. En Francisco Morazán se utilizaron quintiles debido a que hay un mayor número de municipios (27 municipios en estudio) que en el Departamento de Yoro (11 municipios en estudio).

Este estudio estuvo exento de revisión por el comité de ética, porque consistió en la recopilación y el análisis de datos ya existentes y de acceso público, en los cuales no puede identificarse a las personas individualmente.

\section{RESULTADOS}

En 27 municipios del departamento de Francisco Morazán se registraron 71 menores de cinco años fallecidos en el periodo de estudio, de los cuales 30 eran mujeres y 41 hombres. El mayor número de niños que fallecieron procedían del municipio de Curaren (6 hombres y 4 mujeres), seguido de Lepaterique (6 hombres y 3 mujeres), y la Villa de San Francisco (2 hombres y 4 mujeres). La Villa de San Francisco presento una tasa de alfabetismo de $85 \%$ de habitantes mayores de 15 años y tasa de mortalidad de $7 \times 1000$ nacidos vivos que es la más alta en Francisco Morazán. El municipio de Alubaren presento la tasa más baja de alfabetismo: $63 \%$ de habitantes mayores de 15 años, y una tasa de mortalidad de los menores de cinco años de $4 \times 1000$ nacidos vivos. (Ver cuadro1).

En los municipios del departamento de Yoro, se registraron 131 menores de cinco años fallecidos. El número más alto de los niños que fallecieron procedían del municipio de Olanchito (13 hombres y 12 mujeres). El municipio de Yorito registró la tasa más baja de alfabetismo y una tasa más alta de mortalidad 
del menor de cinco años de $6 \times$ cada 1,000 nacidos vivos. El municipio de El Progreso tiene la tasa más alta de alfabetismo, $90 \%$ de población mayor de 15 años y la tasa de mortalidad de $1 \times 1000$ nacidos vivos. (Ver cuadro 2)

Los rangos de edad de los fallecidos menores de cinco años en los 27 municipios del departamento de Francisco Morazán se situaron con más frecuencia en el rango de edad de $0 \mathrm{a}$ 12 meses ( 33 hombres y 26 mujeres.) El grupo poblacional en el departamento de Yoro correspondió a un rango de edad de 0 a 12 meses (48 hombres y 58 mujeres). El índice de Kuznets para el departamento de Francisco Morazán mostro la diferencia absoluta de desigualdad de 1.7 más muertes de menores de cinco años $X$ cada 1000 nacidos vivos en el quintil menos alfabetiza-

Cuadro 1. Niños menores de cinco años fallecidos según sexo, tasa de mortalidad, tasa alfabetismo de 27 municipios del departamento de Francisco Morazán Honduras, Año 2014

\begin{tabular}{|c|c|c|c|c|}
\hline \multirow[t]{2}{*}{ Municipio } & \multicolumn{2}{|c|}{ sexo } & \multirow{2}{*}{$\begin{array}{c}\text { Tasa } \\
\text { Mortalidad }^{a} \\
\text { X1000 }\end{array}$} & \multirow{2}{*}{$\begin{array}{c}\text { Tasa } \\
\text { alfabetismo } \\
\text { x100 }\end{array}$} \\
\hline & Mujer & Hombre & & \\
\hline Curaren & 4 & 6 & 5 & 69 \\
\hline Orica & 2 & 3 & 4 & 76 \\
\hline Guaimaca & 3 & 3 & 2 & 84 \\
\hline El Porvenir & 1 & 3 & 2 & 78 \\
\hline Alubaren & 1 & 1 & 4 & 63 \\
\hline Talanga & 0 & 3 & 2 & 86 \\
\hline Santa Lucia & 0 & 2 & 2 & 92.5 \\
\hline San Antonio de oriente & 1 & 3 & 1 & 88 \\
\hline Tatumbla & 0 & 1 & 5 & 86 \\
\hline Marale & 0 & 1 & 1 & 64 \\
\hline Lepaterique & 3 & 6 & 3 & 76 \\
\hline La Libertad & 0 & 0 & 0 & 77 \\
\hline San Miguelito & 0 & 1 & 5 & 77 \\
\hline La Venta & 1 & 0 & 2 & 78 \\
\hline San Juan de Flores & 0 & 1 & 1 & 79 \\
\hline Maraita & 1 & 0 & 2 & 80 \\
\hline Vallecillo & 1 & 1 & 3 & 81 \\
\hline San Ignacio & 0 & 0 & 0 & 82 \\
\hline Ojojona & 1 & 2 & 3 & 83 \\
\hline Cedros & 2 & 0 & 1 & 83 \\
\hline Nueva Armenia & 1 & 0 & 4 & 84 \\
\hline Sabanagrande & 1 & 0 & 1 & 85 \\
\hline Reitoca & 1 & 1 & 2 & 67 \\
\hline Villa de San Francisco & 4 & 2 & 7 & 85 \\
\hline San Buenaventura & 1 & 0 & 3 & 88 \\
\hline Valle de Ángeles & 0 & 1 & 1 & 90 \\
\hline Santa Ana & 1 & 0 & 2 & 91.2 \\
\hline Total fallecidos & 30 & 41 & & \\
\hline
\end{tabular}

Fuente: Base de datos de mortalidad de niños menores de cinco años. SESAL. 2014. Instituto Nacional de estadísticas Honduras 2013.

a Población menor de cinco años ${ }^{\mathrm{b}}$ Habitantes mayores de 15 años. do; y la brecha relativa de desigualdad es 2 veces mayor en el grupo con menor tasas de alfabetismo (Ver Figura 1A). En el departamento de Yoro la diferencia absoluta de desigualdad es de 1 muerte más $X 1000$ nacidos vivos para el cuartil más alfabetizado; y la brecha relativa de desigualdad es 2 veces mayor en el grupo con tasas más altas de alfabetismo (Ver Figura 2A).

Al realizar la medición con el Índice de Desigualdad de la Pendiente en la mortalidad de los niños menores de cinco años del departamento de Francisco Morazán, este fue de 12.94 $31.04=-18.1$ puntos porcentuales. En este caso, hay un exceso de 18 muertes de niños menores de cinco años por cada 1,000 nacidos vivos entre la población con menor porcentaje de alfabetismo en comparación con la población con mayor concentración de alfabetismo. (Ver Figura 1B). En el departamento de Yoro el índice de desigualdad de la pendiente en la mortalidad de los menores de cinco años correspondió a 13.91 - $37.09=$ -23.18 , lo que indica un exceso de 23 muertes de niños menores de cinco años por cada 1,000 nacidos vivos entre la población con menor concentración de alfabetismo en comparación con la población con mayor concentración de alfabetismo (Ver Figura 2B).

La medición de la desigualdad utilizando el índice de concentración de la salud para el departamento de Francisco Morazán la curva de concentración de la salud se mantiene sobre la línea de equidad (línea roja), por lo que la mortalidad de los niños menores de cinco años se concentra en la población menos alfabetizada (Ver Figura 1C). En el departamento de Yoro la curva de concentración de la salud se mantiene sobre la línea de equidad, por lo que la mortalidad de los menores de cinco años se concentra en la población menos alfabetizada.

Cuadro 2. Niños menores de cinco años fallecidos según sexo, tasa de mortalidad, tasa alfabetismo de 27 municipios del departamento Yoro Honduras, Año 2014

\begin{tabular}{lcccc}
\hline \multicolumn{1}{c}{ Municipio } & \multicolumn{2}{c}{ sexo } & $\begin{array}{c}\text { Tasa } \\
\text { Mortalidad } \\
\text { X1000 }\end{array}$ & $\begin{array}{c}\text { Tasa } \\
\text { alfabetismo }^{b} \\
\text { X100 }\end{array}$ \\
\hline Olanchito & 12 & 13 & 2 & 87 \\
Yoro & 8 & 8 & 2 & 79 \\
El Progreso & 10 & 6 & 1 & 90 \\
Victoria & 10 & 6 & 5 & 80 \\
Morazán & 7 & 9 & 4 & 80 \\
Negrito & 5 & 6 & 2 & 82 \\
Yorito & 3 & 9 & 6 & 76 \\
Sulaco & 7 & 2 & 5 & 80 \\
Santa Rita & 4 & 1 & 3 & 88 \\
Arenal & 3 & 0 & 2 & 86 \\
Jocon & 2 & 0 & 5 & 79 \\
Total & 71 & 60 & & \\
fallecidos & & & & \\
\hline
\end{tabular}

Fuente: Base de datos de mortalidad de niños menores de cinco años. SESAL. 2014. Instituto Nacional de estadísticas Honduras 2013.

a Población menor de 5 años, ${ }^{b}$ Habitantes mayores de 15 años 
Figura 1. Desigualdades en la mortalidad en menores de cinco años. Departamento de Francisco Morazán, Honduras, 2014.

\section{A. Índice de Kuznets (Brecha absoluta y relativa)}

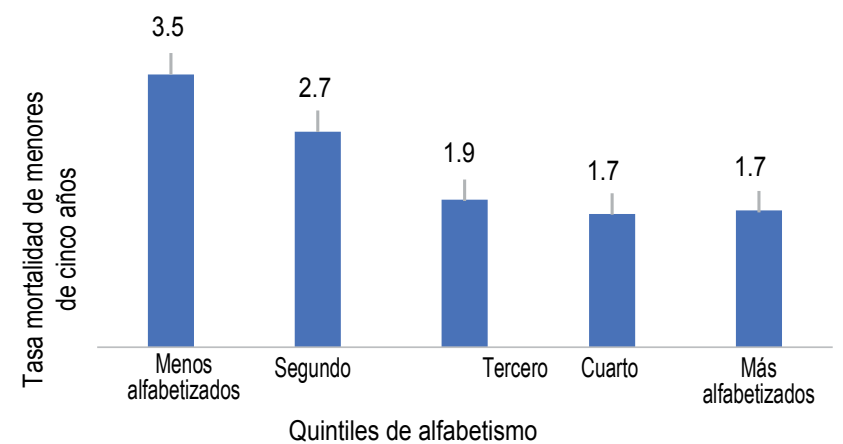

B. Índice de desigualdad de la pendiente.

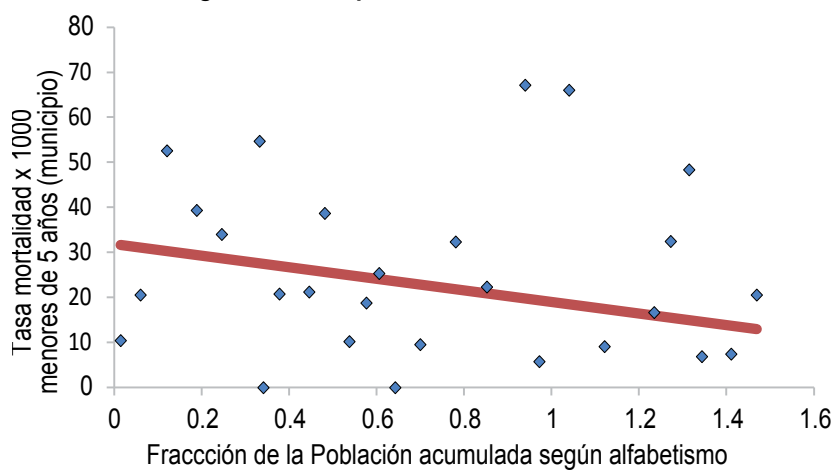

C. Índice de concentración de la desigualdad en salud.

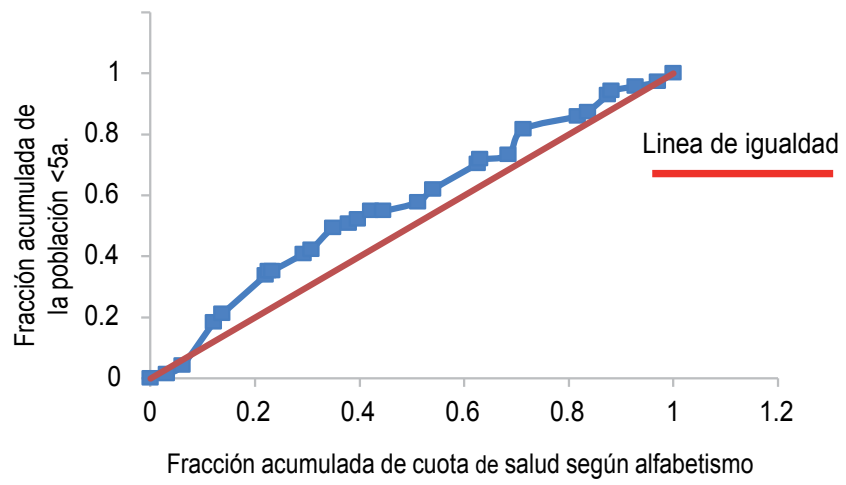

La curva de concentración y la línea de equidad se alinean en el punto $0.2005,0.2137$ que corresponde al municipio de Yoro, esto quiere decir que no se marca una diferencia entre el alfabetismo y mortalidad; significa que hay equidad entre estas dos variables ya que este municipio tiene de las tasas más bajas de alfabetismo y de las más bajas de mortalidad (Ver Figura 2C).

\section{DISCUSIÓN}

A medida que las agendas contemporáneas más relevantes de la salud pública mundial y regional se van alineando en sus componentes conceptuales y emerge más explícitamente el rol medular de la equidad, va creciendo también el recono-
Figura 2. Desigualdades en la mortalidad en menores de cinco años. Departamento de Yoro, Honduras, 2014.

A. Índice de Kuznets (Brecha absoluta y relativa)

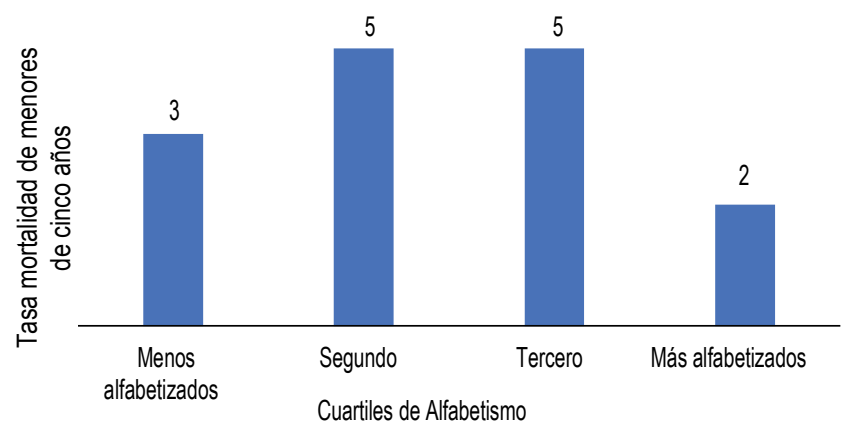

B. Índice de desigualdad de la pendiente.

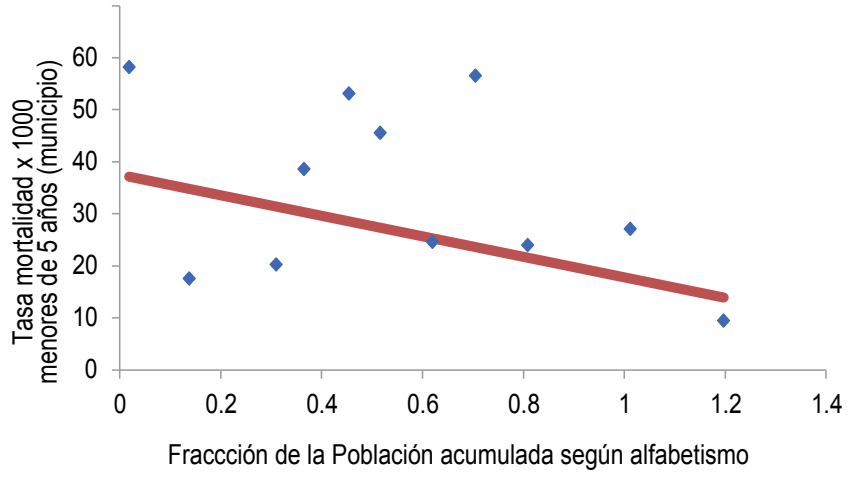

C. Índice de concentración de la desigualdad en salud.

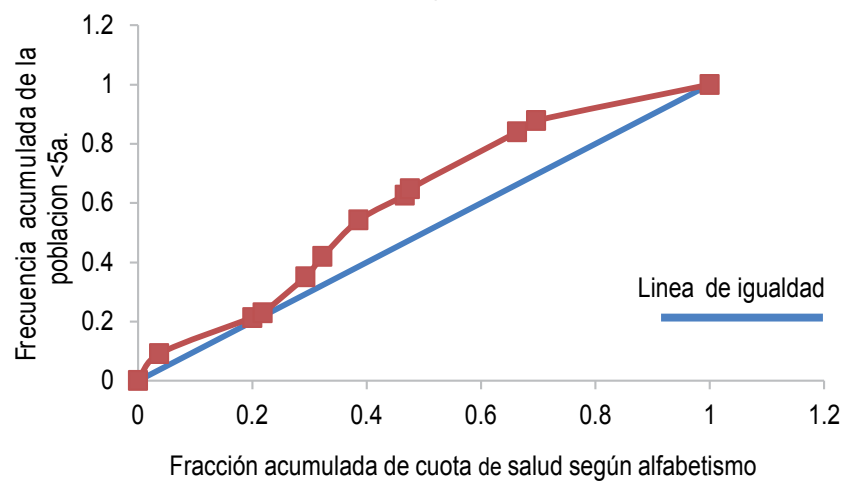

cimiento del valor estratégico del monitoreo de desigualdades sociales en salud como el instrumento por excelencia de la inteligencia sanitaria para juzgar objetivamente el progreso hacia la equidad en salud. ${ }^{14,15} \mathrm{La}$ orientación en brindar los datos de salud en base a promedios nacionales a reducido la atención en la disminución de las desigualdades sociales, las cuales han venido siendo documentada cada vez con mayor insistencia por la investigación desde el ámbito académico y no desde el ente rector de la salud en el país.

La caracterización demográfica de los niños menores de cinco años muestra que en el departamento de Yoro existe mayor población que en los 27 municipios de Francisco Morazán; en ambos departamentos la edad en que fallecen la mayor cantidad de niños es en el primer año de vida. 
La mortalidad de los niños menores de cinco años en los departamentos de estudio se desplazó considerablemente hacia los grupos poblacionales con niveles educativos más bajos. En el departamento de Francisco Morazán el quintil más alto de acuerdo con el alfabetismo se situó en municipio de Santa Lucia, seguido por Santa Ana y Valle de Ángeles. Estos municipios también tienen las tasas de mortalidad más bajas. El quintil más bajo de acuerdo con el alfabetismo correspondió a los municipios de Reitoca, Marale y Alubaren, con tasas de mortalidad entre 1 a 4 x 1000 nacidos vivos. Casi invariablemente, aquellos que ocupan posiciones más bajas en la escala socioeconómica exhiben peor estado de salud que quienes están por encima de ellos en la jerarquía, cuanto más alto sea el posicionamiento social, mejor será la salud. Las sociedades más pobres y los sectores más pobres de la población en las sociedades tienen consistentemente tasas de mortalidad más altas y esperanza de vida más baja que sus contrapartes menos pobres. ${ }^{14,16}$

En cuanto al departamento de Yoro, el cuartil más alto de salud de acuerdo con el alfabetismo correspondió al municipio de El Progreso y Santa Rita; estos tienen tasas de mortalidad entre 1 y 2 x 1000 nacidos vivos. Es importante mencionar que el municipio de Progreso se encuentra ubicado contiguo al departamento de Cortés, por lo que posiblemente esta población tiene oportunidades de acceso a los servicios educativos y de salud de dicho departamento que es una zona de alto desarrollo económico y social del país. El Cuartil más bajo correspondió a los municipios de Yorito, Yoro y Jocón; El municipio de Yorito tiene la tasa más alta mortalidad del menor de cinco años: $6 \mathrm{x}$ cada 1000 nacidos vivos. La educación tiene un efecto directo en algunos determinantes de la mortalidad, la mortalidad del niño por ejemplo está influida por las creencias y valores que la madre tenga sobre el cuidado de su hijo y el comportamiento ante sus enfermedades. ${ }^{17}$

Una investigación que evidencia y cuantifica la desigualdad educativa observada en Honduras, a través de un análisis comparativo por departamento y género fue realizada en 2015 , utilizando la base de datos de la Encuesta Permanente de Hogares de Propósitos Múltiples (EPHPM) 2009 y 2011, encontró que la desigualdad educativa medida por el coeficiente de Gini, varía entre regiones, para el caso Francisco Morazán obtuvo un coeficiente Gini 0.282 y Yoro un coeficiente Gini de 0.308 en el año 2011, ubicando a Yoro en uno de los departamentos que más desigualdades presenta en años de escolaridad por departamento. ${ }^{18}$

Behn et al ${ }^{17}$ han analizado el riesgo de morir entre el nacimiento y los dos años en 13 países latinoamericanos en función de la educación de la madre; los países tienen niveles de mortalidad muy diferentes, pero en todos ellos se observa que el riesgo de morir del niño en esa edad desciende de modo marcado a medida que la educación de la madre aumenta. De este modo, los hijos de mujeres analfabetas tienen un riesgo de morir que es de 3.5 a 5 veces mayor que los de mujeres que han alcanzado diez y más años de educación. En Bolivia la mortalidad entre los recién nacidos de las mujeres sin educación supera a las 100 defunciones por 1000 nacidos vivos, en cambio la mortalidad entre los recién nacidos con madres con al menos educación secundaria es menor de 40 defunciones por 1000 nacidos vivos. ${ }^{19}$

En los 27 municipios de Francisco Morazán, la diferencia absoluta entre la tasa de mortalidad de niños menores de cinco años del grupo con el quintil más bajo y el grupo con el quintil más alto es de 2 muerte por cada 1,000 niños menores de cinco años nacidos vivos. En términos relativos, la tasa de mortalidad de niños menores de cinco años en el grupo con el menor nivel de educación es dos veces mayor que la tasa de mortalidad de niños menores de cinco años en el grupo con el mayor nivel de educación.

En el departamento de Yoro, la brecha más alta de desigualdad se encuentra en el primer cuartil, que representa a los municipios con menor tasa de alfabetismo y corresponde a los municipios de Yorito, Yoro y Jocon. El cuartil más alto corresponde a los municipios de Sulaco, Victoria y Yoro. La brecha absoluta indica que hay 1 muerte por cada 1000 nacidos vivos en los municipios menos alfabetizados en relación con los municipios más alfabetizados; al igual que la brecha relativa. Una de la desventaja de medir desigualdades con brechas es que solo toman en cuenta los extremos. ${ }^{4}$ En Yoro los municipios que están situados en el segundo y tercer cuartil tienen mayores brechas de desigualdad en la mortalidad de la niñez.

Un sólido conjunto de indicadores, tales como el índice de concentración y el índice de desigualdad de la pendiente, se han utilizado para cuantificar el grado de desigualdad en salud asociado con la jerarquía social definida por una escala de ingreso u otras variables de estado socioeconómico. Se ha demostrado tempranamente un efecto umbral con la pobreza, así como otras medidas ominosas de privación material (tales como analfabetismo, falta de agua potable y saneamiento, hambre $o$ incluso falta de acceso a la atención de la salud).,311,15

El Índice de Desigualdad de la Pendiente (IDS) en la mortalidad de los niños menores de cinco años del departamento de Francisco Morazán fue de -12.85 (redondeado es igual a 13). El signo negativo indica que conforme se avanza en la escala social, la mortalidad de los menores de cinco años disminuye. En este caso, hay un exceso de 13 muertes de niños menores de cinco años por cada 1,000 nacidos vivos entre la población con menor concentración de alfabetismo. El IDS en el Departamento de Yoro correspondió a -0.20 , lo que significa que hay un exceso de 20 muertes de niños menores de cinco años entre la población con menor concentración de alfabetismo.

En el departamento de Francisco Morazán el Índice de Concentración de la Salud fue igual a -0.14 , indica que la mortalidad de la niñez se concentra menos en los municipios más alfabetizados (indicado por el signo negativo). Al igual en el departamento de Yoro el Índice de Concentración de la Salud es igual a -0.2 , indicando que la mortalidad de la niñez se concentra menos en los municipios más alfabetizados (indicado por el signo negativo).

Por medio de los resultados obtenidos, se observa que la desigualdad en la mortalidad de los niños menores de cinco años, que persiste más desigualdad en el departamento de 
Yoro que en Francisco Morazán, de acuerdo con el estratificador alfabetismo.

Para lograr los Objetivos de Desarrollo Sostenible aplicado a Honduras específicamente el área estratégica de intervención número uno: una Honduras sin pobreza extrema, educada y sana, con sistemas consolidados de previsión social, ${ }^{20}$ es necesario un monitoreo que incluya la medición de las desigualdades sociales para observar los avances en la salud de las poblaciones excluidas y el acceso a servicios de salud integrales y de calidad, desde el abordaje del derecho a la salud. Avanzar de manera progresiva, hacia la prioridad nacional (Plan de Nación 2010-2022) de cobertura universal y gratuita de los servicios de salud, priorizando a las poblaciones más vulnerables y así evitar las desigualdades sociales de la salud. Hablar de salud y educación es estrechar dos variables que están realmente ligadas al desarrollo social. Para que en Honduras se brinde el derecho a la salud a los niños menores de cinco años, se debe dar educación a sus pobladores en general de una manera igualitaria y equitativa.

La brecha más alta de desigualdad de mortalidad en la niñez en ambos departamentos está en los municipios que se encuentran en el quintil más bajo de educación; correspondiendo a los municipios de Marale, Reitoca, Curaren, Alubaren para Francisco Morazán y los municipios de Yorito, Sulaco y Victoria para el departamento de Yoro. Los resultados de las métricas de desigualdad que se aplicaron en este estudio concluyen que, en los 27 municipios de Francisco Morazán y los municipios del departamento de Yoro, se observó que los municipios con tasas más altas de alfabetismo tienen menos mortalidad de menores de cinco años, que los municipios con tasa de alfabetismo bajo, que son los que tienen mayor mortalidad en menores de cinco años.

La realización de la medición de desigualdades sociales en salud tiene sus limitaciones y están relacionadas con la revisión de la información disponible ya que esta no es sistemática ni completa en las categorías de análisis a nivel de municipios, aun así, los datos disponibles permiten llegar a algunas conclusiones importantes. Hace falta institucionalizar el monitoreo de desigualdades en salud como parte constitutiva del ejercicio de la primera función esencial de salud pública: el análisis de la situación de salud y sus tendencias..$^{15,21}$

Las limitaciones en esta investigación estuvieron asociadas a la dificultad de poder conocer los datos de variables como nivel de alfabetismo a nivel de municipio; los datos sociodemográficos que recoge el INE no llegan a representar los valores a este nivel.

"La desigualdad en la salud es la peor de las desigualdades. No hay peor desigualdad que saber que morirás antes por ser pobre." Frank Dobson

\section{CONTRIBUCIONES}

Ambas autoras concibieron y diseñaron el estudio. NCRC lideró la conducción de la investigación. EGGG lideró la recolección y análisis de la información. NCRC lideró la redacción del artículo. Ambas autoras aprobaron la versión final del artículo.

\section{AGRADECIMIENTO}

Se agradece al Dr. Bomar Méndez Rojas, consultor OPS/ OMS Tegucigalpa, por sus aportes en la revisión de la investigación.

\section{REFERENCIAS}

1. Mújica OJ, Moreno CM. De la retórica a la acción: medir desigualdades en salud para "no dejar a nadie atrás. Rev Panam Salud Pública. 2019;43:e12.

2. Hernández Oré MA, Sousa LD, López JH. Honduras desatando el potencial económico para mayores oportunidades. Diagnóstico sistemático de país. Washington D.C.: Banco Mundial; 2015.

3. Reno R, Hyder A. The Evidence base for social determinants of health as risk factors for infant mortality: a systematic scoping review. J Health Care Poor Underserved. 2018;29(4):1188-1208.

4. Organización Panamericana de la salud. Manual para el monitoreo de las desigualdades Washington, D.C.: Organizacion Panamericana de la Salud; 2016

5. De la Hera A B, Roncero U M. Desigualdades sociales en la salud de la población de la comunidad autónoma del país vasco. Vitoria Gasteiz: Ararteko, Graficas Santa María; 2007.

6. Barreto ML. Desigualdades en Salud: una perspectiva global. Ciênc saúde coletiva [Internet]. 2017[citado 23 mayo 2020];22(7):20972108. Disponible en: https://www.scielo.br/scielo.php?pid=S141381232017002702097\&script=sci_abstract\&tlng=es

7. Haeberer M, Noguer I, Mújica OJ. Desigualdades educacionales en mortalidad y supervivencia de mujeres y hombres de las Américas, 19902010. Rev Panam Salud Pública. 2015;38(2):89-95.

8. Augsburger AC, Gerlero S, Galende S, Moyano CB. La expresión de las desigualdades sociales en la mortalidad infantil. Información epidemiológica en regiones seleccionadas de la provincia de Santa Fe (Argentina). Rev Fac Nac Salud Pública 2013;31(supl 1): S139-S148.

9. Instituto Nacional de Estadísticas (HN). XVII Censo de poblacion y VI de vivienda a nivel de municipios. Tegucigalpa: INE; 2013.

10. Instituto Nacional de Estadísticas (HN). Indicadores de población. [Internet]. 2018. Tegucigalpa: INE; 2018. [citado julio 2018]. Disponibleen: www.ine.gob.hn

11. Servizo Galego de Saúde. Medición de desigualdades de salud [Internet]. España: Sergas; 2014 [citada 21 marzo 2018]. Disponible en: https://www.sergas.es/Saude publica/Documents/1901/Ayuda_Epidat4_ Medicion_de_desigualdades_en_salud_Octubre2014.pdf

12. Schneider MC, Castillo Salgado C, Bacallao J, Loyola E, Mújica O, Vidaurre $\mathrm{M}$, et al. Métodos de medición de desigualdades de salud. Rev Panam Salud Pública. 2002;12(6):398-415.

13. Every Woman Every Child Latin America and Caribbean. Guía paso a paso para el cálculo de métricas de desigualdad en salud. Panamá: EWC-LAC; 2016.

14. Mújica OJ. Cuatro cuestiones axiológicas de la epidemiología social para el monitoreo de la desigualdad en salud. Rev Panam Salud Pública. 2015;38(6):433-41.

15. Hosseinpoor AR, Bergen N, Koller T, Prasad A, Schlotheuber A, Valentine $\mathrm{N}$, et al. El monitoreo orientado a la equidad en el contexto de la cobertura universal de salud. PLoS Med. 11(9): e1001727.

16. Duarte Gómez MB, Núñez Urquiza RM, Restrepo Restrepo JA, Richardson-López-Collada VL. Determinantes sociales de la mortalidad infantil en municipios de bajo índice de desarrollo humano en México. Bol Med Hosp Infant Mex [Internet]. 2015[citado 17 diciembre 2020];72(3):181189.

17. Behm H. Determinantes económicos y sociales de la mortalidad en América Latina. Salud Colectiva. 2011;7(2):231-253. 
18. Espino I. Desigualdad educativa en Honduras: un análisis comparativo por departamento y género. Documento de trabajo. Buenos Aires: CLACSO; 2015.

19. Organización Mundial de la salud. Comisión de determinantes sociales de la salud. Subsanar las desigualdades en una generación: alcanzar la equidad sanitaria actuando sobre los determinantes sociales de la salud: resumen analítico del informe final.[Internet]. Washington D.C.: OMS; 2008. [consultado 14 mayo 2020]. Disponible en: https://apps.who.int/iris/ handle/10665/69830

20. Organización de Naciones Unidas. Marco de Asistencia de las Naciones Unidas para el desarrollo: Honduras 2017 - 2021. Tegucigalpa: ONU; 2016.

21. Mújica OJ. [Editorial]. Trascendiendo la invisibilidad: el monitoreo de las desigualdades sociales en salud. Hacia promoc. salud. 2016;21(1):9-11.
ABSTRACT. Background: Social inequalities in health refer to health disparities, which are considered unfair, avoidable, and unnecessary, and which systematically affect vulnerable populations. Objective: Measure social inequalities in health of mortality in children under five years of age, Francisco Morazán and Yoro, Honduras, using 2014 data. Methods: Using a crosssectional descriptive approach, this study analyzed the mortality of children under age five through the lens of social inequality measurement and analyzed the sociodemographic characteristics of the deceased. In addition, this study estimated relevant health inequality indicators: the relative and absolute Kuznets index, the slope inequality index, and the health concentration index. It also examined inequalities in mortality across municipal literacy rate. Results: There were a total of 71 registered deaths of children under age five in Francisco Morazán, whereas there were 131 registered in Yoro. An excess of 18 deaths of children under five per 1000 live births was found among the population with the lowest literacy in Francisco Morazán, and 23 in Yoro, compared to the municipalities with the highest literacy. In both departments, the health concentration curve remained close to the equity line, leading to the conclusion that under-five mortality among children was concentrated among the municipalities with the lowest literacy rate. Discussion: Municipalities with the highest literacy rates were observed to have lower mortality rates for children under age five.

Keywords: Health inequalities, Mortality, Inequity concentration Index. 